\title{
Prevention of transformers' damage in HPP with double generating units connected to GIS via HV cables
}

\author{
V. Silbermann \\ Department of Electrical Systems and Networks, \\ Fichtner Consulting Company, Germany
}

\begin{abstract}
Many hydro power plants (HPP) in various countries are being operated with double generating units (DGU) where each unit generator-transformer has its own generator circuit breaker (CB) and both units are connected to a high voltage (HV) network via two common CBs installed in an air-insulated switchgear (AIS). Such circuitry was designed to halve the number of rather expensive $\mathrm{HV} \mathrm{CBs}$ and to save on the space necessary for their installation. The necessary flexibility of HPP is continued as DGUs allow keeping one unit transformer in idle mode and putting into operation a unit generator via generator CB very rapidly if necessary. Longterm experience has demonstrated the high reliability of operation of HPPs with such circuitries, nevertheless due many reasons the AISs in HPPs are replacing now with more compact and convenient in operation gas-insulated switchgears (GIS). This replacement is taking place in many countries, and everywhere, the existing DGUs are connected to GISs via HV cables. It shall also be noted that each GIS circuitry is generally designed identically to previous AIS circuitry. But cases of damage of some unit transformers working in idle mode have been observed in a number of countries after such replacement. This paper reports the results of investigating one of these cases of damage, discusses the possible cause of these incidents and elaborates some measures to prevent such damage in the future.
\end{abstract}

Keywords: GIS, HV cable, idle mode of transformer, magnetizing characteristic of transformer core, Rayleigh area, resonance in $L-R-C$ circuit. 


\section{Introduction}

An increase in the number of cases of transformers' damage has been observed in many countries over the last thirty years, and as a general rule, the damaged transformers were connected to gas insulated switchgears (GIS) via high voltage (HV) cables. Data coming from various countries demonstrate that 27 of 1,133 such transformers with rated voltages from $50 \mathrm{kV}$ up to $800 \mathrm{kV}$ have been damaged. The results of damage analysis of these transformers presented in reference [1] testify about over voltage risk of transformers which are connected to GIS via HV cables. Practically all these failures were caused during typical switching operations like operations in substations described in [2]. Windings were damaged in six transformers, the tap changer - in one, insulators - in nineteen transformers and one time the damage of the tapped transformer connected to the unit transformer was fixed.

An analysis of the conditions accompanying these cases of damage led to the conclusion that over voltages generated in transient conditions in electric power networks were the reason for defects in many power transformers which passed the standardized electric withstand tests with a positive result. And all damaged transformers were protected against over voltages in the line with standard IEC recommendations and their protection equipment has not pickup nowhere. It can therefore be concluded that this damage was caused by so-called very fast transient over voltages (VFTOs) [2].

In accordance with [1] and [2] these VFTOs appear in fact by any switching operations in GIS where all equipment is connected to the bus bars via HV cables.

These VFTOs have been investigated and described in many papers (e.g. in [3, 4]), but all these investigations have limitations as far as clarifications and descriptions of parameters of transient over voltages themselves are concerned. And only in [3] it is mentioned that this damage appeared at transformers working in idle mode. Usually the idle mode of transformers is used in hydro power plants (HPP) that are being operated with double generating units (DGU) where each unit generator-transformer has its own generator circuit breaker $(\mathrm{CB})$ and both units are connected to a high voltage (HV) network via two common CBs. Such operations provide the necessary flexibility of HPP where the generator can be synchronized via transformer at idle within some minutes if necessary. Because of that the analysis of processes by switching operations in GISs and elaboration on measures to prevent the damage of transformer at idle while retaining the HPPs' flexibility of is a relevant objective.

\section{Description of investigated damage}

The HPP where the analysing damage occurred consists of nine generators with an apparent power $325 \mathrm{MW}$. Six of them get hooked up via $400 \mathrm{MVA}, 15 / 500 \mathrm{kV}$ generator transformers and $500 \mathrm{kV} \sim 600 \mathrm{~m}$ single cables to GIS $500 \mathrm{kV}$ and three of them get hooked up via 400 MVA, $15 / 220 \mathrm{kV}$ generator transformers and $220 \mathrm{kV} \sim 500 \mathrm{~m}$ single cables to GIS $220 \mathrm{kV}$. Both GISs are connected via two 501 MVA, 500/220/10 kV autotransformers. The GIS $500 \mathrm{kV}$ and GIS $220 \mathrm{kV}$ 
have been replaced the corresponding AISs with analogous circuitries. The 500 $\mathrm{kV}$ part of electrical scheme of this HPP is shown in Fig. 1, where GIS equipment is shown within dotted blue box and all damaged elements are shown in red. The protection of corresponding elements against over voltage are performed by means of standard surge arresters $\mathrm{Zn} 0$ Class 4 with $\mathrm{U}_{\mathrm{c}}=581 \mathrm{kV}$.

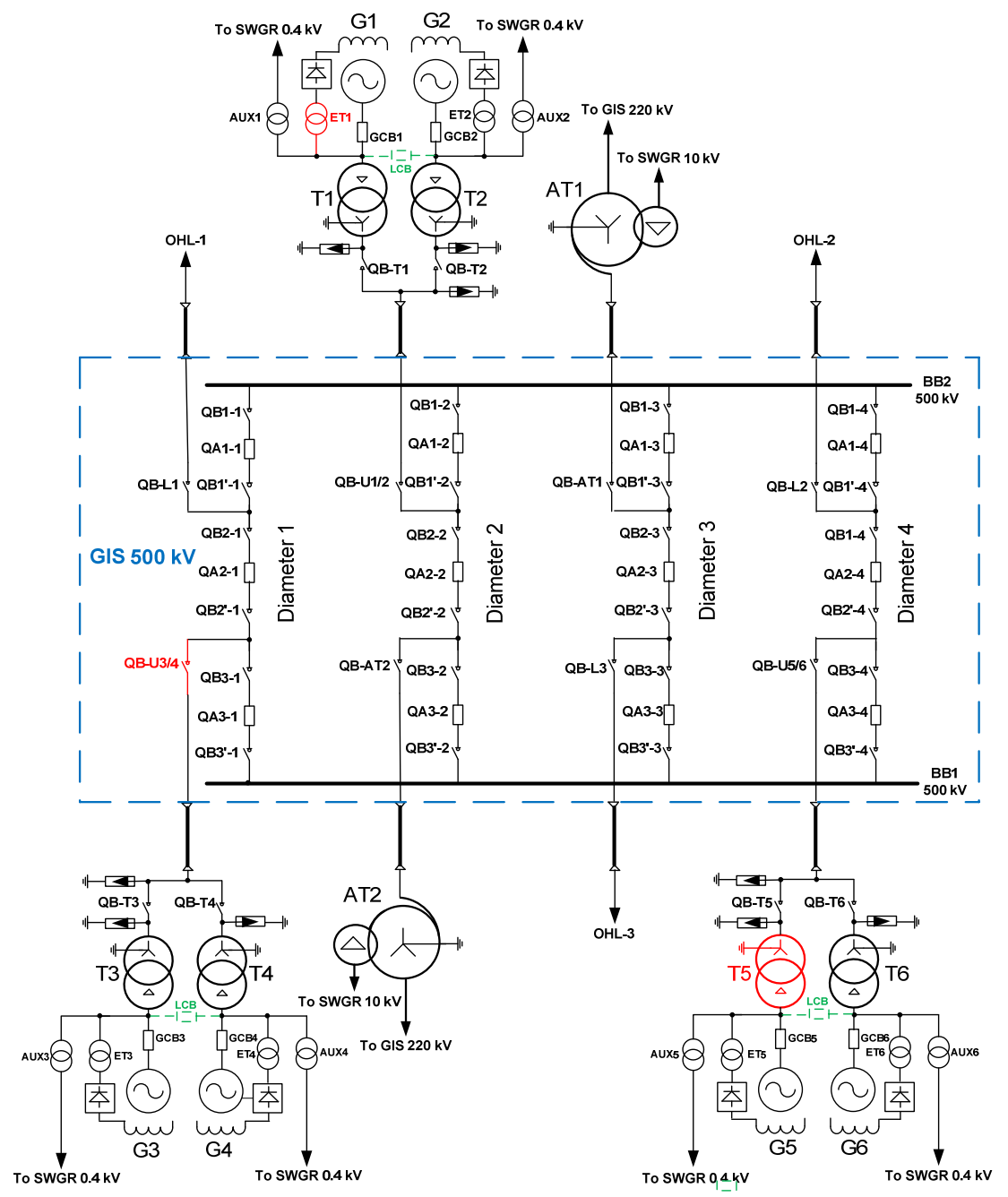

Figure 1: Single line diagram of electrical part $500 \mathrm{kV}$ of HPP.

It was preliminarily scheduled that the control system of disconnectors QB-T3 and QB-T4 has to be updated and tested. Both disconnectors are installed in the switchyard behind the HPP building. The shutdown of the $500 \mathrm{kV}$ components at the DGU 3/4 (cable, DS and associated equipment) is necessary for this purpose. 
This work was scheduled during autumnal minimum of load. Because of this, during the commutations the generator transformers $\mathrm{T} 1$ and $\mathrm{T} 5$ were operated in idle mode (the disconnectors QB-T1 and QB-T5 were closed and the generator CBs GCB 1 and GCB5 were open).

After successful switching off of units 3 and 4 by the generator CBs GCB3 and GCB4 incl. the switching off their disconnectors QB-T3 and QB-T4, the switching of the disconnector QB-U3/4 in the $500 \mathrm{kV}$ GIS supplying the disconnectors QBT3 and QB-T4 via cable line was necessary. While switching off this disconnector (QB-U3/4) in the $500 \mathrm{kV}$ GIS manually the following happened simultaneously:

- Transformer T1 which was operated at idle was tripped by the transformer differential protection and also by current cutoff protection of tapped transformer ET1. With this the whole DGU $1 / 2$ was shut down. It was found that the tapped exciter transformer ET1 $(630 \mathrm{kVA}, 15.75 /$ $0.4 \mathrm{kV}$ ) was irreparably damaged (the high voltage connections of this transformer to the unit G1-T1 were melted and disconnected from the unit). Generator transformer T1 was also checked by insulation tests, but it was found to be undamaged and put back into operation again, together with Generator 1 and the new exciter transformer.

- Transformer T5 was tripped by the transformer differential protection. With it the whole DGU 5/6 was shut down. It was found that the phase "A" of its HV winding was irreparably damaged, and it was out of operation.

- Taking into account that the generators G3 and G4 were already switched off, the supply of all OHLs $500 \mathrm{kV}$ outgoing from GIS $500 \mathrm{kV}$ was continued only from generators in GIS $220 \mathrm{kV}$ via autotransformers AT1 and AT2.

- An excessive noise was also noted during operation of the disconnector QB-U3/4 and SF 6 gas leakage was detected in the compartment (phase "A") of this disconnector. But the pressure ( 3.8 bar) remained above the alarm level. Therefore, a shut-down of this disconnector was not necessary.

- Only one of the surge arresters $(\mathrm{Uc}=581 \mathrm{kV})$ at the side $500 \mathrm{kV}$ of the DGU 2 was operated during this incident.

The analysis the data saved in the event and disturbance recorders in corresponding relays in GIS $500 \mathrm{kV}$ revealed that:

- All HPP's protection equipment responded and tripped absolutely correctly during the investigated event.

- All over voltage magnitudes fixed by recorders in the protection relays (about $580 \mathrm{kV}$, i.e. at the boundary of pick-up of surge arresters which explains why only one of many surge arresters was operating) were located within the admissible margins of over voltages measured by FAT tests of both damaged transformers. Such over voltages themselves should not lead to the damage of excitation transformer ET1 and generator transformer $\mathrm{T} 5$. 
- $\quad$ But it shall be also emphasized that recording of the parameter of VFTO during this event was factually not possible due to the restriction of the frequency range of relay recorders to the limit of $10 \mathrm{kHz}$, just as these frequencies may exceed 2-2.5 $\mathrm{MHz}$ [4].

\section{Formulating of a task of investigation (analysis)}

It shall be preliminary noted that a similar operation with another disconnector of cable line (QB-U5/6) performed in the summer when all HPP generators (and generator transformers correspondingly) were loaded did not lead to any disturbances in the HPP operation. Thus the following issues shall be analyzed and clarified:

- Why were only the transformer at idle itself and the equipment connected to the transformer at idle damaged although over voltages branched throughout in whole GIS and their values recorded in many points of GIS were equal.

- Is the operation of disconnectors under voltage in GIS with connected cable lines allowed or should all such operations be performed only with circuit breakers.

- What can be done to minimize the risk of appearance of VFTOs in GIS generally and by commutations in particular.

The purpose of this paper is to discuss the specialties of appearance of VFTOs by commutations in the GIS with cable lines, estimation their parameters and their influence on the insulation of equipment of HPP. Measures that could reduce the probability of the appearance of such over voltages without essential increase in cost for HPP are being in operation will also be discussed.

The deliberations will be based on records and measuring made during the event described above and their comparison with the data recorded during the investigations described in [3] and [4].

\section{The clarification of processes in a transformer by its operation at idle discussion}

Taking into consideration that only transformers at idle or the equipment working together with such transformers were damaged by commutations, it appears worthwhile to examine the magnetizing characteristics of power transformers to clarify the non-linearity of process in transformer by its operation at idle. A typical transformer magnetizing characteristic is shown in Fig. 2. This magnetizing characteristic of the transformer core until its "knee" consists of two essential parts: the well-known practical linear segment in the presence of medium and high magnet fields (from 0.2 to 1.2 Tesla) and the so called Rayleigh area in the presence of low magnet fields (from 0 to 0.2 Tesla). The transformer inductivity in the linear segment of the magnetizing characteristic of the transformer core is likewise linear, i.e. the magnetic induction is described by formula 
where:

$$
\mathrm{B}=\mu_{0} * \mathrm{H}+\mathrm{M}
$$

B - magnetic induction (in Tesla);

$\mathrm{H}$ - magnetic field strength (in $\mathrm{A} / \mathrm{m}$ );

$\mu_{0}$ - magnetic constant of core material;

$\mathrm{M}$ - resultant magnetic dipole moment per unit volume of core material, which is constant for each core material.

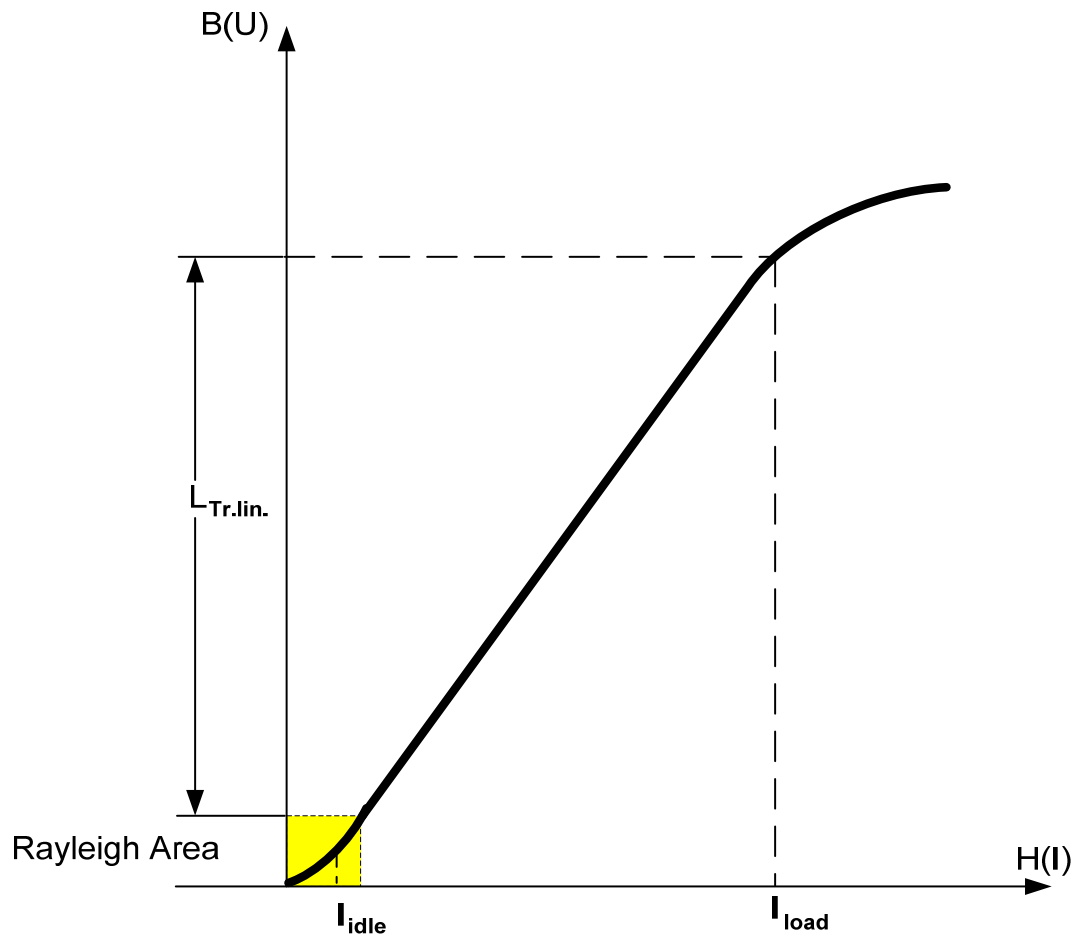

Figure 2: Typical magnetizing characteristic of power transformer.

The Rayleigh Area (yellow field) is an area of low magnetic fields of magnetizing characteristic of the transformer core. The behaviour of ferromagnetic materials in this area is described by the Rayleigh law, i.e.

where:

$$
\mathrm{B}=\chi_{0} * \mathrm{H}+\alpha \mathrm{R} * \mu 0 * \mathrm{H}^{2}
$$


$\chi_{0}-$ initial magnetic susceptibility, i.e. a measure of the magnetic properties of a core material. It indicates whether a core material is attracted into or repelled out of a magnetic field;

$\alpha \mathrm{R}$ - Rayleigh constant, describes the irreversible properties of a magnetic material;

For the transformer sheets the Rayleigh area covers the segment of the magnetizing characteristic till the magnetic field strength takes on the value 0.01 $\mathrm{A} / \mathrm{m}$, i.e. until the currents in the $500 \mathrm{kV}$ winding about $5 \mathrm{~A}$. This means that the idle currents of unit transformers (about $2 \mathrm{~A}$ on the side $500 \mathrm{kV}$ for $400 \mathrm{MVA}$ transformers) are located within Rayleigh area, and the transformer inductivity will be essential non-linear in accordance with (2). Consequently, the L-R-C circuit consisting of cable capacity and the transformer at idle will be non-linear as well. Taking into account that high frequency over voltage impulses will arise during any commutation of preliminary charged HV cable [2] and that these impulses will be branched out through whole GIS, the processes in the L-R-C circuit consists of capacity of $500 \mathrm{kV}$ cable, non-linear inductivity and resistance of transformer at idle and the grounding system of HPP will, in principle, be oscillating. Theses oscillating processes that arise along with commutations in GIS can also lead to the resonances in each non-linear L-R-C circuit. Unfortunately, as pointed out in [3] the consideration of the processes taking place inside the winding is a difficult task, as it is impossible to access windings of operating equipment. Obtaining the required parameters by calculation is also extremely difficult because of the complexity of the calculations and the lack of data on the windings and insulation system geometry. Hence these oscillating processes can only be estimated qualitatively. However, it is possible to measure the frequency characteristics of transformers' windings to determine the resonance frequencies and the magnitudes of VFTOs peaks. Such measurements for the block transformer 25 MVA, 110/15 kV were performed and studied in [4] and it was shown that by introducing such VFTOs in the transformer winding their magnitude can be quadruple of the voltage at the transformer terminals. And if it is even assumed that due to resonance in this L-R-C circuit the magnitude of over voltage, that arose during the investigated event, was double the value recorded at the transformer terminals, the voltage magnitude within the transformer winding will exceed $1160 \mathrm{kV}$, i.e. the level of the impulse test voltage. And the issue is exacerbated by the fact that the shape of these over voltages measured by research in Russia [3] differs substantially from the standard wave of test voltage and the slope of voltage curve can attain $450 \mathrm{kV} / \mu \mathrm{s}$. This greatly exceeds the standard lightning impulse voltage of $1-5 / 50 \mu$ s as specified in [5] and it is also above the highest impulse voltage level for $500 \mathrm{kV}$ equipment of $1550 \mathrm{kV}$ peak as stipulated in [6].

Consequently, it can be ascertained that the resonant over voltages with high slope of voltage curve in the windings of transformers $\mathrm{T} 1$ and $\mathrm{T} 5$ arose during commutation of disconnector QB-U3/4 and these acted on the weak points in this equipment (first of all in the winding insulation). For the relatively new 
transformer T1 (in operation since 2011) this weak point was the tapped exciter transformer ET1 (in operation since 1979); in the case of transformer T5, which has been in operation since 1976, it was the transformer itself.

It has also to be noted in this connection that although over voltage impulses appearing during commutation of preliminary charged cable are propagated and spread throughout all parts of the HPP primary circuits and grounding system connected together, such VFTO peaks cannot arise, if transformer is operating under load, i.e. in the linear part of its magnetization characteristic as such transformer together with the charged cable and ground resistance makes up a nonresonant L-R-C circuit.

\section{Possible measures to prevent the transformers' damage: discussion}

As it may be concluded from the above analysis the idle mode of generator transformers should be excluded to prevent their possible damage by VFTOs (over voltages). Of course the most comprehensive solution would be the modification of HPP circuitry to separate the unit's generator-transformer. Operation of generator transformers at idle would, in principle, be excluded while retaining the existing flexibility by synchronising of the unit's generator-transformer at the 500 $\mathrm{kV}$ side. But this is a very expensive solution that would require duplication of HV cables and with regard to circuitry shown in Fig. 1 the arrangement of one additional diameter consisting of three additional HV circuit breakers. To avoid such high expenditure alternatives are discussed beginning with simplest solutions.

1. As shown above VFTOs in the circuitries like that depicted in Fig. 1 only arise during switching actions (on and off) on such L-R-C circuits by operation of any transformer at idle. It means that the performance of necessary switching actions (on and off) on such L-R-C circuits by circuit breaker would avoid the occurrence of the VFTO peaks to a great extent due to its short opening time (approx. $20 \mathrm{~ms}$ ). Disconnectors in GIS have an opening time of about $500 \mathrm{~ms}$. This means that the probability of transient voltage damage during opening the circuits by circuit breaker is about 25 times lower if tis done using a disconnection switch. Hence all switching actions in $500 \mathrm{kV}$ cable circuits with or without idle running transformers (both on and off) shall be performed only by circuit breakers as a general measure. The cable disconnectors may be operated only when the corresponding cable is discharged. The fast earthing switches in $500 \mathrm{kV}$ GIS should be used for this discharging.

2. The operation philosophy of all HPPs with DGU should be checked with regard to the possibility of keeping all unit generator transformers loaded, i.e. the possibility of operating the generators loaded in the range of 50 $100 \mathrm{MW}$. 
3. If this is not be possible, the switching operations on any cable with disconnectors in the GIS at "on" position should be made only after disconnecting all transformers at idle. Upon completion of operation with cable disconnectors the corresponding transformers can be energized with their disconnectors installed in the switchyard behind the HPP building again resulting the idle mode.

4. It can be also recommended that additional load circuit breakers (LCB) would be installed between LV windings of DGU transformers (shown in Fig. 1 as dotted green lines) if the breaking capacity of the existing generator circuit breakers allows such an increase of short circuits values at LV side of these transformers, should be installed. These LCBs shall be switched on in the DGU with one transformer at idle before any commutation in HV circuitry of HPP and switched off upon accomplishment of these commutations.

\section{Conclusions}

1. The appearance of very fast resonance transient voltages during commutations in the CIS with cable lines is unavoidable if one of the transformers connected to GIS operates at idle.

2. The magnitudes of these over voltages within transformers at idle may essentially exceed the standard values applied during the testing of these transformers and they can lead to the breakdown of transformer insulation and irreparable transformer damage.

3. To prevent such damage, the operation of any generator transformer at idle during commutation in the HPP HV circuitries should either be excluded or the duration of these commutations should be minimized.

4. Some measures for exclusion of operation of transformers at idle are proposed in this paper for the discussion.

\section{References}

[1] E. E. Henriksen, "Study of Very Fast Transients Over Voltages in Transformers" Electra, No. 179, pp. 13-23, 1998.

[2] SIGRE Working Group 33/13.09 - "Very fast transient phenomena associated with GIS"- CIGRE Paper 33-13/1988 Session.

[3] Yu. A. Lavrov, A. G. Ovsyannikov, S. S. Shevchenko, O. Yu. Shiller, "Over Voltage at Switching of $500 \mathrm{kV}$ Power Block Transformer with SF6 Circuit Breaker". Electro, No. 6, pp. 24-27, 2010 (in Russian).

[4] M. Florkowski, J. Furgal. "Terminal based recognition of resonance over voltages in transformer windings". Proceedings of the 16-th International symposium on High Voltage engineering, Innes House, Johannesburg, 2009. - Paper A1).

[5] IEC Standard IEC 60060-1.

[6] IEC Standard IEC 60071-1. 